EPiC Series in Computing
Volume 56, 2018, Pages $84-92$
Proceedings of the 5th International
OMNeT+ + Community Summit

\title{
An Integrated Framework for Fog Communications and Computing in Internet of Vehicles
}

\author{
Alessio Bonadio, Francesco Chiti, and Romano Fantacci \\ University of Florence, Florence, Italy \\ \{name.surname\}@unifi.it
}

\begin{abstract}
In this paper, the novel Fog Communications and Computing paradigm is addressed by presenting an integrated system architecture, that is applied to achieve a full context awareness for vehicular networks and, consequently, to react on traffic anomalous conditions. In particular, we propose to adopt a specific co-designed approach involving Application and Networks Layers. For the latter one, as no infrastructure usually exists, effective routing protocols are needed to guarantee a certain level of reliability of the information collected from individual vehicles. As a consequence, we investigated classical Epidemic Flooding based, Network Coding inspired and Chord protocols. Besides, we resort to Blockchain principle to design a distributed consensus sensing application. The system has been tested by resorting to OMNeT++ framework for its modularity, high fidelity and flexibility. Performance analysis has been conducted over realistic scenarios in terms of consensus making overhead, latency and scalability, pointing out the better trade-off allowing the overlay P2P network formation and the complete context awareness achieved by the vehicles community.
\end{abstract}

\section{Introduction}

In recent years, the Cloud Computing (CC) paradigm [1] has become very popular by providing an ubiquitous on-demand network access to remote computing and storage platforms, or even services, that can be rapidly provisioned with minimal design and operational effort. However, several constraints in terms of requested bandwidth and real time processing suggest to move processing as close as possible to data generation units so as to minimize the "data production to decision making" latency.

In this perspective, Fog Computing (FC) is an emerging paradigm that extends CC towards the edge of the network [9], where applications/services run directly at the network edge in smart end-devices. The goal of FC is to improve efficiency and reduce the amount of data that needs to be transported to the Cloud for massive data processing, analysis and storage. Furthermore, the design of efficient solutions within FC also requires investigate a novel communication/networking paradigm, called Fog Networking (FN), in order to meet specific configurability, adaptability, flexibility and energy/spectrum-efficiency constraints. Despite FN 
exhibits promising technical features, new challenging issues are raised; in particular, significant research efforts have to be devoted for integrating efficient and functional networking and computing capabilities in a same $\mathrm{FC}+\mathrm{FN}$ platform.

The "Green Adaptive Fog Computing and Networking Architecture" (GAUChO) project aims, indeed, at designing a novel distributed and heterogeneous architecture able to functionally integrate and jointly optimize FC and FN capabilities in the same platform. The joint $\mathrm{FC}+\mathrm{FN}$ architecture, representing the overall outcome of the project, aims at supporting lowlatency and energy-efficiency as well as security, self-adaptation, and spectrum efficiency by means of a strict collaboration among end-devices. Specifically, Task T1.3 aims at proposing new methodologies to support the network formation phase, allowing fixed or mobile devices (mainly vehicles) to be connected, so as to achieve a full context-awareness by means of exchanging and jointly refining context-related information. The focus is on designing novel topology-control protocols that dynamically track any context change (e.g., devices location, traffic patterns, channel quality, quality of active information flows).

From a networking perspective, this vision is strictly related to the so called Vehicular Ad hoc NETworks (VANETs) paradigm, that is a special kind of Mobile Ad hoc NETworks (MANETs) composed by vehicles as well as access points located at the edges of the roads [12]. Recently, the paradigm of Internet of Vehicles (IoV) has been introduced into this domain to provide a wireless communication environment that allows vehicles to exchange traffic-related information [2], while enabling passengers to communicate with a remote Cloud or each other via in-vehicle wireless devices [4]. The latter aspect, where according to FC vision edge devices are involved in in situ data gathering, dissemination and processing, is a promising method for to fulfil VANETs requirements, e.g., to enhance the reactiveness to sudden context variations and to real-time data analyse [10].

While the actual reference standard for IoVs is represented by IEEE 1609/WAVE (Wireless Access in the Vehicular Environment), with both vehicle-to-vehicle (V2V) and RSU-to-vehicle $(\mathrm{R} 2 \mathrm{~V})$ [14] interfaces, VANETs are expected to rely on an abstract and flexible communication mode, which is usually referred as vehicle-to-everything (V2X) $[6]^{1}$.

IoV could be particularly beneficial in the fields of traffic safety and management, since vehicles often utilize broadcast of traffic-related information (such as vehicle position, speed, hazards) and other services to maintain traffic safety. Moreover, this could be effectively supported through cooperative applications, where a group of vehicles could jointly optimize the driving process efficiency. In particular, vehicles participating in a cooperative application are expected to make coordinated and mutually consistent decisions. To ensure consistency, it is often essential that the participating vehicles reach agreement on the data they use as a basis for these decisions. In this perspective, we applied the blockchain (BC) principle, that was originally conceived as a digital payment framework. It enables a unambiguous ordering of transactions to guarantee the integrity and consistency of the blockchain across geographically distributed nodes. When applied to VANET realm, BC has to take into account some specific challenges, mainly, the fast time varying topology. As a consequence, adaptable and robust mobile architectures are needed [5, 8]. To face these issues, we proposed here an integrated approach, managing the network topology set-up and maintaining, together with the information dissemination and validation in a decentralized and autonomous way. In particular, it involves the investigation of state-of-the-art routing approaches, from classical Delay Tolerant Networking (DTN) oriented schemes, up to massive Network Coding (NC) and Chord approaches, to achieve a cooperation gain which reduces the consensus making latency at the increasing of

\footnotetext{
${ }^{1}$ Several technologies supporting this approach have been proposed within fifth generation (5G) mobile communication systems, among which Cellular-V2X from 3GPP R14.
} 

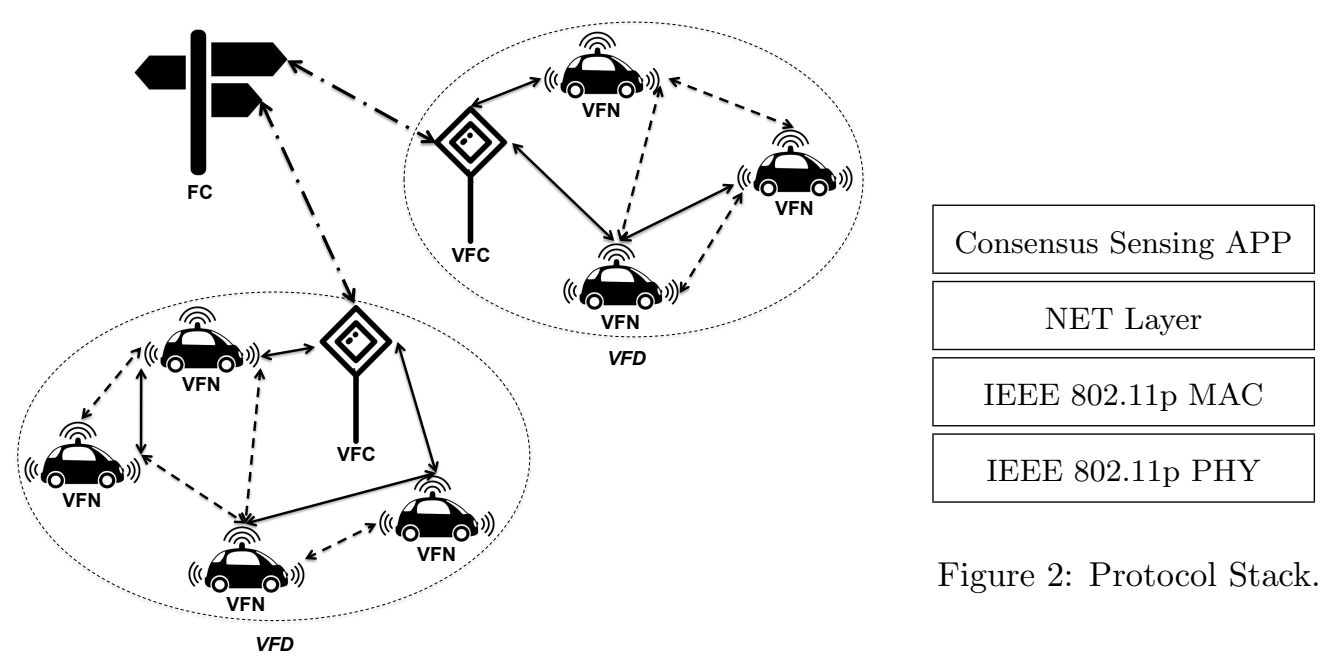

Figure 2: Protocol Stack.

Figure 1: Vehicular Fog overall architecture.

network size and vehicles (dis)connections rate due to mobility.

To effectively support the validation of the overall designed system, we resort to OMNeT++ simulation framework, as he provide a state-of-the-art standardized protocols implementation and a modular and flexible approach to integrated additional modules.

The rest of this paper is organized as follows. In Section 2, we characterised the joint $\mathrm{FN}+\mathrm{FC}$ platform with specific reference to BC oriented applications for VANET, providing an insight into the development over OMNeT++ framework. This allowed to derive Section 3 several performance metric that pointed out the effectiveness of the proposed approach in terms of scalability, reactiveness and overhead. Finally the conclusions are drawn in Section 4.

\section{Integrated OMNeT++ oriented Framework}

The reference Vehicular Fog (VF) architecture considered for our proposal is represented in Figure 1, where two VF Domains (VFDs) comprised of VF Nodes (VFNs) and VF Controllers (VFCs), are depicted. In addition logical (i.e., related to the overlaying application) and physical (i.e., specific to the underlying network) communications interfaces are represented with dotted or solid lines, respectively. A Fog Controller (FC) has been also introduced to allow the interoperability among VFDs.

The VFN functional model, adopted in designing our integrated approach, is shown in Fig. 2, that is comprised of:

- Consensus Sensing (CS) Application designed according to BC technology;

- Network Layer functionalities, as defined below;

- Physical and Data Link Layers compliant with IEEE 802.11p specifications. 
It can be noticed that Transport Layer functionalities are not included in the proposed architecture as usual in VANETs; therefore, our application directly uses services provided by NET Layer.

As a reference scenario, we consider a typical traffic congestion, characterized by slowing speeds and vehicular queuing. This usually occurs in the presence of a red traffic light or even due to an accident. In this situation, it is particularly relevant that the involved vehicles quickly achieve context awareness by means of distributed data collection and fusion about the $\mathrm{road} /$ traffic conditions. Thus, vehicles are requested to (i) arrange the pool into a network and then (ii) start the CS application, as shown in Figure 2. Further, this enables a distributed decision process, relaying on the information generated by each vehicle.

The proposed architecture has been modeled with reference to OMNeT++ environment, as it fully supports IEEE $802.11 \mathrm{p}$ standard through a comprehensive suite of models included in its Veins framework (which could be extended towards $5 \mathrm{G}$ system via VeinsLTE). This allows our proposal to be aligned with the de facto standard for automotive industry, to accurately model the vehicular domain and to test its performance by means of network simulations as realistic as possible.

\subsection{Consensus Sensing Application}

According to the reference scenario previously introduced, vehicles are expected to achieve a full context awareness by means of a distributed data gathering procedure. This requires also a local information reconciliation by applying a CS application, that has been designed according to the BC technology. As a matter of fact, this allows all the participants to read from a distributed ledger, that records all the observations from vehicles. The derived chain is updated using a protocol, which guarantees a common view of the overall information. Moreover, it assures the integrity and the consistency of the ledger and its non ambiguous ordering.

We developed the application starting from the Proof of Work (PoW) mechanism, which has been modeled with a uniform random distribution, as in real systems the delay introduced by PoW is usually upper limited. We also set the block size $B$, representing the amount of information to be validated as a whole. In particular, we perform a validation via the joint consensus of at least half of the network nodes, so $B=N / 2$, where $N$ is the number of nodes.

Finally, we implement two new message types:

- ObservationMessage (OM), which extends WaveShortMessage and carries the information collected by a vehicle;

- ValidationMessage (VM), which is a WaveShortMessage and contains the validation of an information block.

Once the network is formed, all vehicles send the information they collected by the sensors with OMs. All nodes update the content of their block as information is received. Once a vehicle fills a block, it initiates the validation phase and, after a random delay, sends the validated block to other nodes using a VM. The procedure starts again with the next block.

\subsection{Routing Protocols}

Before starting CS application, vehicles are requested to form a network; this is dictated by the need of quickly achieving a distributed decision, which considers all the local information. To this purpose, several routing protocols to provide Network Layer functionalities have been investigated and optimized to our use case, as presented in the following. 


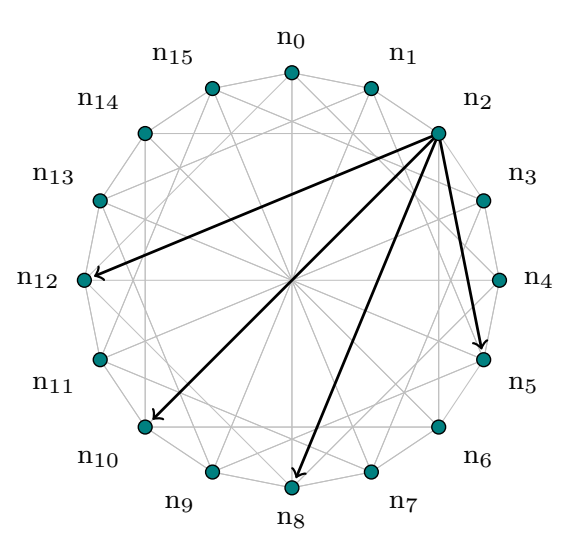

Figure 3: Chord's network topology.

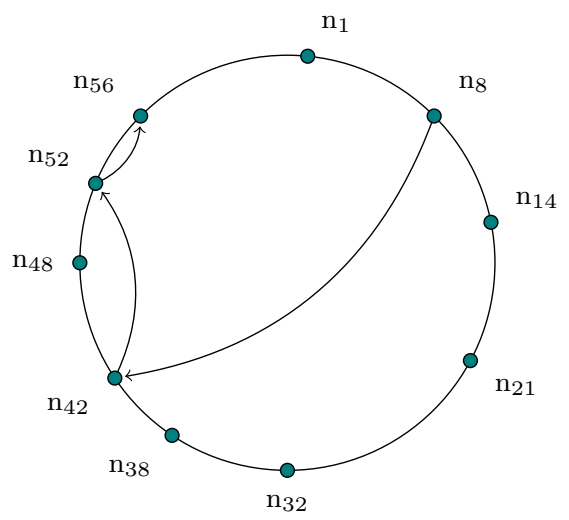

Figure 4: Chord's lookup.

DTN oriented approach. Delay Tolerant Network (DTN) paradigm enable transfer of data over links that may lack continuous connectivity. For this reason, the purpose of many existing DTN routing protocols is to increase the likelihood of finding a path between sender and receiver [3]. Data dissemination in VANETS is performed with DTN protocols usually divided into two main families [13]: (i) Epidemic protocols, representing the simplest approach and (ii) Geographic protocols, which are based on nodes location. We focus on Epidemic protocols, as their inherent anycast addressing scheme is better suited to the characteristics of CS applications. Specifically, three specific techniques are investigated:

- Blind Flooding (BF), i.e., the simplest version of epidemic protocol, where each node forwards the received message to all its neighbors;

- TTL-based Flooding (TF), that limits the retransmission of a message according to a Time To Live (TTL) counter;

- Probability-based Flooding (PF), each node retransmits the message to its neighbors with a probability $P$, with $0<P<1$.

Network Coding. Another effective protocol investigated in this paper for information block dissemination to a community of vehicles relies on the generalized multiflow Network Coding (NC) principle [7]. It enhance the basic DTN approach by allowing each device to store, carry and forward a random linear combination of the previously received packets. In particular, we adopted an epidemic DTN routing joined with a fountain code to limit the complexity and overhead without requiring any topology information, and effectively exploiting the nodes mobility by iterate the basic scheme.

Chord. This approach is based on distributed hash tables (DHT), used to realize a decentralized peer-to-peer (P2P) overlay network [11], as shown in Figure 3. Chord provides the mapping of keys into nodes, used to pursue the resolution between L2 and L3 addresses. Chord is extremely efficient in lookups resolving. Indeed, it needs only $O(\log N)$ messages [11] to reach any node in the network, as it depicted in Figure 4. Moreover, each node maintains information only about $O(\log N)$ other node, so that join and leave events only requires no more than 


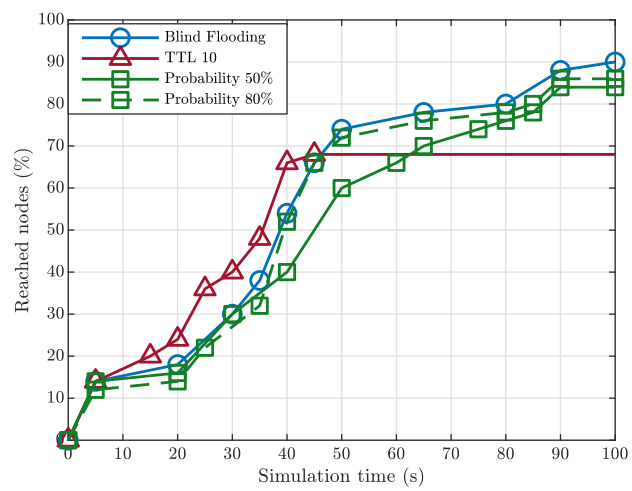

Figure 5: DTN, reached nodes.

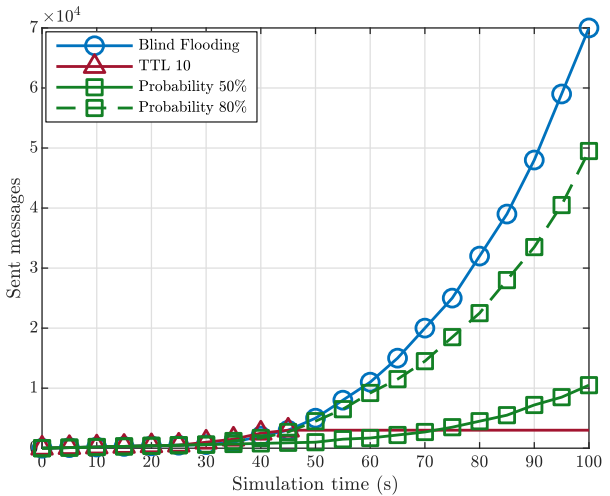

Figure 6: DTN, sent messages.

$O\left(\log ^{2} N\right)$ messages [11]. When integrated in our framework, Chord provides a dynamic and distributed address resolution table, which adapts to join and leave events.

\section{Performance Evaluation}

The proposed integrated approach has been developed and tested via OMNeT++ framework for achieving high fidelity modeling and performance evaluation. We first focus on the Network layer to eventually take into account the overall system figures.

\subsection{DTN oriented approach}

The scenario under investigation is a grid plan city, whose map has been imported from Open Street Map. When the simulation starts an accident occurs, and the damaged vehicle has to inform the surrounding ones (which we limited to 50 cars).

This simulation is realized using Veins' Car and RSU modules. The communication between nodes is provided by Nic80211p, which allow them to send and receive information via WaveShortMessages. We also set the playground as a square of $10 \mathrm{~km} \times 10 \mathrm{~km}$, and the length of the simulation to $100 \mathrm{~s}$.

We start with analyzing the percentage of reached cars in Figure 5. In particular TF based technique presents the worst performance, reaching about $70 \%$ of the nodes, and stopping after about $45 \mathrm{~s}$. This is due to the fact that it often inhibits retransmissions, while $\mathrm{BF}$ and $\mathrm{PF}$ approaches improve the performance almost in the same way.

In addition to this, the protocol overhead, i.e., the number of sent messages, is pointed out in Figure 6, where the differences among the various approaches are more remarkable. In particular, it is evident the advantage of PF methods, as they requires less overhead to achieve the same percentage of alerted vehicles. It is worth noting that adopting $P=0.5$ the number of sent messages is about $1 / 7$ of the ones needed by $\mathrm{BF}$. 


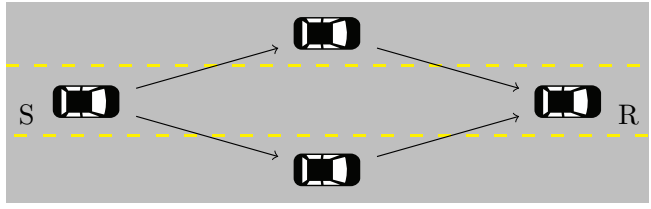

Figure 7: Network Coding, test topology.

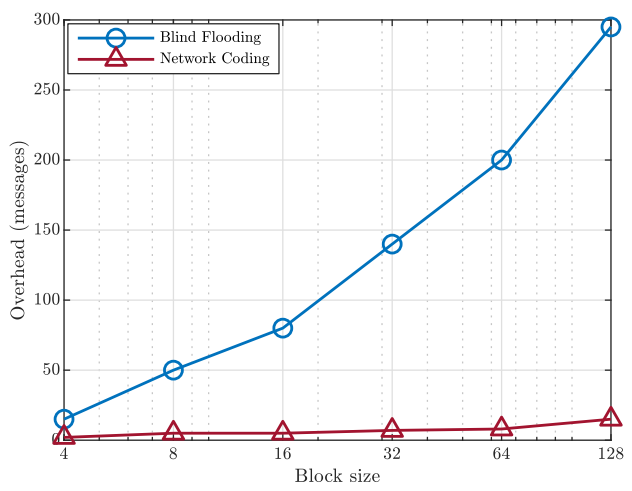

Figure 8: Network Coding, overhead.

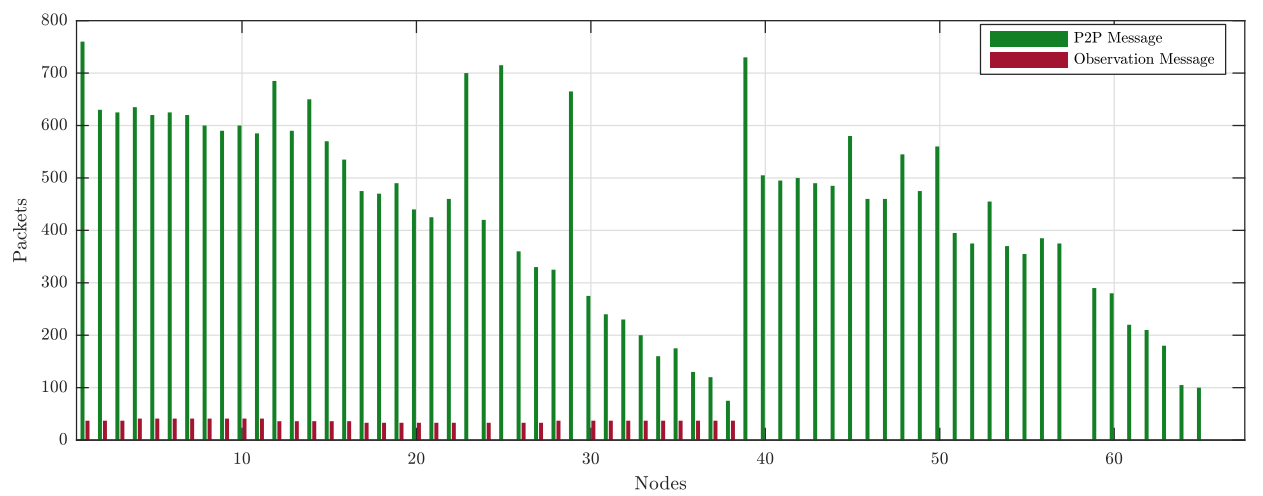

Figure 9: Chord, packets sent.

\subsection{Network Coding}

For evaluating the performance of $\mathrm{NC}$ technique we focus on the typical diamond topology shown in Figure 7, with two Relay nodes between Sender (S) and Receiver (R). The Relay nodes can only perform store, combine and forward of each received packet.

This simulation is realized relying on an external library, Eigen, which manages the coding and decoding of messages. Given the complexity of these phases, we necessarily had to limit the number of nodes. In this scenario we used a module defined from scratch, while messages are WaveShortMessages.

In Figure 8 we can observe the overhead introduced by $\mathrm{NC}$; in this particular scenario, $\mathrm{NC}$ is very efficient. The gap w.r.t. $\mathrm{BF}$ is remarkable and increases at the increasing of packet block size, thank to a kind of diversity gain provided by the two independent Relays.

\subsection{Chord}

In order to evaluate the integrated framework, we adopted a more realistic map (i.e., the default Erlangen map provided by SUMO mobility simulator), with realistic traffic patterns. This 
scenario is realized using Car and RSU modules from Veins and the communication is provided by Nic80211p. To build and maintain the Chord network consistency we also implement a new message type, P2PMessage (P2PM), extending WaveShortMessage. In our simulation the network is composed on the average by 35 cars and it stops when the validation of a specific block is reached. In fact, this condition allows to establish vehicles communities are established that can communicate with each other according to the Small-World Network paradigm.

As shown in Figure 9, we evaluate the overall number of packets sent by each node. Here we consider only messages P2PMs and OMs, which are the most relevant in our scenario. We can observe the presence of two different networks formed, wherein nodes present the same tendency. This is due to the signaling of Chord protocol: for each node joining the network, some routing information needs to be updated. In particular, we can observe that the first nodes to join the network are those that send more messages, whilst the overhead gradually decreases for the other ones. We can also point out that the first community completed the network forming process, and its nodes start to send OMs. The other group has not yet formed, as the simulation is programmed to stop when the first block is filled. Moreover, the number of P2PMs is much higher than OMs, this highlights that the formation of the Chord network is the more critical phase.

In conclusion, it could be pointed out that the number of messages per vehicles needed to disseminate a block of information is roughly equal to $2 \cdot 10^{3}, 10^{3}, 10^{2}, 2 \cdot 10^{2}$, for BF, DTN, $\mathrm{NC}$ and Chord, respectively, but the latter always allows a reliably data distribution, thus representing the best candidate.

\section{Conclusions}

This paper presents an integrated system architecture inspired to the join FN and FC paradigm, applied to achieve a full context awareness for VANET and, consequently, to react on traffic anomalous conditions. In particular, we propose to adopt a specific co-designed approach involving Application and Networks Layers. For the latter one, we investigated classical DTN Flooding based, $\mathrm{NC}$ multiflows and Chord protocols, while we resort to $\mathrm{BC}$ technology to achieve a distributed consensus. The system has been tested by resorting to OMNeT++ and Veins frameworks for their modularity, high fidelity and flexibility. Although the three methods are used in slightly different scenarios, interesting data can still be extracted. Performance analysis pointed out that DTN Probability-based methods is in charge of reaching a significant percentage of cars, without requiring excessive overhead. On the other hand, NC protocols are an interesting alternative to the previous ones, with considerably better performance, even in a scenario where only 4 cars are involved, even in the presence of packet loss. Finally, where Chord scheme is adopted good performance is pointed out in terms of consensus making overhead, while the higher cost is due to $\mathrm{P} 2 \mathrm{P}$ network formation via Chord protocol which in turns allows a highly efficient and resilient topology control.

\section{Acknowledgments}

This work has been supported by the project "GAUChO - A Green Adaptive Fog Computing and Networking Architecture" funded by the MIUR Progetti di Ricerca di Rilevante Interesse Nazionale (PRIN) Bando 2015 - grant 2015YPXH4W-004. 


\section{References}

[1] S. Abolfazli, Z. Sanaei, E. Ahmed, A. Gani, and R. Buyya. Cloud-based augmentation for mobile devices: Motivation, taxonomies, and open challenges. IEEE Commun. Surveys Tuts., 16(1):337368, Jan 2014.

[2] K. M. Alam, M. Saini, and A. E. Saddik. Toward social internet of vehicles: Concept, architecture, and applications. IEEE Access, 3:343-357, 2015.

[3] A. Balasubramanian, B. Levine, and A. Venkataramani. Dtn routing as a resource allocation problem. SIGCOMM Comput. Commun. Rev., 37(4):373-384, Aug 2007.

[4] C. Bila, F. Sivrikaya, M. A. Khan, and S. Albayrak. Vehicles of the future: A survey of research on safety issues. IEEE Trans. Intell. Transp. Syst., 18(5):1046-1065, May 2017.

[5] A. Dorri, M. Steger, S. S. Kanhere, and R. Jurdak. Blockchain: A distributed solution to automotive security and privacy. IEEE Commun. Mag., 55(12):119-125, Dec 2017.

[6] X. Duan, Y. Liu, and X. Wang. Sdn enabled 5g-vanet: Adaptive vehicle clustering and beamformed transmission for aggregated traffic. IEEE Commun. Mag., 55(7):120-127, 2017.

[7] U. Lee, J.-S. Park, J. Yeh, G. Pau, and M. Gerla. Code torrent: Content distribution using network coding in vanet. In Proceedings of MobiShare '06, pages 1-5, New York, NY, USA, 2006. ACM.

[8] A. Lei, H. Cruickshank, Y. Cao, P. Asuquo, C. P. A. Ogah, and Z. Sun. Blockchain-based dynamic key management for heterogeneous intelligent transportation systems. IEEE Internet Things J., 4(6):1832-1843, Dec 2017.

[9] M. Mukherjee, L. Shu, and D. Wang. Survey of fog computing: Fundamental, network applications, and research challenges. IEEE Commun. Surveys Tuts., 2018.

[10] S. A. Soleymani, A. H. Abdullah, M. Zareei, M. H. Anisi, C. Vargas-Rosales, M. Khurram Khan, and S. Goudarzi. A secure trust model based on fuzzy logic in vehicular ad hoc networks with fog computing. IEEE Access, 5:15619-15629, 2017.

[11] I. Stoica, R. Morris, D. Karger, M. F. Kaashoek, and H. Balakrishnan. Chord: A scalable peer-topeer lookup service for internet applications. SIGCOMM Comput. Commun. Rev., 31(4):149-160, Aug 2001.

[12] Y. Toor, P. Muhlethaler, A. Laouiti, and A. D. La Fortelle. Vehicle ad hoc networks: applications and related technical issues. IEEE Commun. Surveys Tuts., 10(3):74-88, March 2008.

[13] S. M. Tornell, C. T. Calafate, J. C. Cano, and P. Manzoni. Dtn protocols for vehicular networks: An application oriented overview. IEEE Commun. Surveys Tuts., 17(2):868-887, Q2 2015.

[14] R. A. Uzcategui, A. J. De Sucre, and G. Acosta-Marum. Wave: A tutorial. IEEE Commun. Mag., 47(5):126-133, May 2009. 